\title{
On the origin of recrystallization textures
}

\author{
K T KASHYAP \\ Central Materials and Processes Laboratory, Hindustan Aeronautics Ltd., Bangalore 560 017, India
}

MS received 10 August 2000

\begin{abstract}
The development of recrystallization textures has been debated for the past 50 years. Essentially the rival theories of evolution of recrystallization textures i.e. oriented nucleation (ON) and oriented growth (OG) has been under dispute. In the ON model, it has been argued that a higher frequency of the special orientation (grains) than random occur, thus accounting for the texture. In the OG model, it has been argued that the specially oriented grains have a high mobility boundary and thus can migrate faster and grow to a larger size as compared to random orientations thus contributing to the final recrystallization texture.

In FCC metals and alloys like aluminium, cube orientation $[(001)\langle 100\rangle]$ is the recrystallization texture component. In the classic OG model, cube orientation is supposed to be misoriented from $S$-orientation $[(123)\langle 63 \overline{4}\rangle]$ which is a deformation texture component by a $40^{\circ}$ about $\langle 111\rangle$ relationship which is supposed to be a high mobility boundary leading to faster growth of cube grains. Stereographic calculations and analytical calculations are presented in this paper to the effect that the $S$-orientation $(123)\langle 63 \overline{4}\rangle$ is not misoriented from cube $(100)\langle 001\rangle$ by $40^{\circ}$ (111) whereas another deformation texture component $(123)\langle 41 \overline{2}\rangle$ which is termed the $R$-component is misoriented from cube component by $40^{\circ}\langle 111\rangle$. $R$-component is also seen in deformation textures of aluminium and hence the classic OG model remains valid with respect to the $R$-component.
\end{abstract}

Keywords. Recrystallization; oriented nucleation; oriented growth; cube orientation

\section{Introduction}

During plastic deformation of metals, the slip imposed crystal rotation of each grain gives rise to the development of deformation textures. Annealing the deformed, textured material leads to a wide range of recrystallization textures. In a review on annealing textures, Grewen and Huber (1978) pointed out that from similar alloys each having almost identical deformation texture, very different annealing textures can be produced. At one extreme a largely retained rolling texture can be produced but on the other hand very sharp new textures of which cube (001) [100] in rolled FCC metals can be produced.

To go into the origins of sharp textures i.e. cube in FCC metals, there are two rival theories to understand the origins dating back 50 years. The first of these ideas 'oriented nucleation (ON)' was discussed by Burgers and Louwerse (1931) and the second idea 'oriented growth (OG)' was discussed by Barret (1940).

The essential idea of $\mathrm{ON}$ is that new grains with the orientation of the major component of the recrystallization texture, for example, the cube orientation in rolled FCC metals, nucleate at a much higher frequency than do grains of all other orientations.

Mackenzie and Thompson (1957) showed that for a cubic crystal symmetry in a random polycrystalline array the frequency of grains within $20^{\circ}$ of a given orientation such as the exact cube is about $4 \%$, therefore the pre- diction of $\mathrm{ON}$ is that the measured number fraction of cube oriented new grains $F_{\mathrm{c}}$ will be

$$
F_{\mathrm{c}} \gg 0.04 \text {. }
$$

This result was confirmed by Samajdhar et al (1994) in a DC cast commercial purity aluminium using electron back scattering patterns technique. The essential feature of $\mathrm{OG}$ is that grains with the required orientation for the recrystallization texture grow faster than grains of other orientations. The prediction of OG can be expressed as

$$
G_{\mathrm{c}}=r G(r \gg \gg 1) .
$$

$G_{\mathrm{c}}$, the growth rate of grains with the recrystallization texture orientation e.g. cube, $G$ the growth rate of grains with random orientation, $r$ the growth rate factor.

Hence it can be supposed that in the case of cube recrystallization texture, cube grains will be larger as opposed to grains with other orientations.

The OG model as supported by Beck and Hu (1966) and Lucke (1984) is the misorientation of the cube component of the recrystallization texture to the $S$-component of the deformation texture (123) $[63 \overline{4}]$ is $40^{\circ}$ about a common rotation axis (111). This misorientation relationship is well known since the work of Aust and Rutter (1965) and Ibe and Lucke (1966) give a high mobility to the grain boundary. 
In this paper calculations are presented to show that cube (001) [100] is not misoriented by $40^{\circ}\langle 111\rangle$ to the

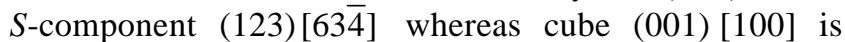
misoriented by $40^{\circ}\langle 111\rangle$ with $R$-component (123) [41 $\left.\overline{2}\right]$ which is also a component in the deformation texture of rolled FCC metals like aluminium.

The idea of OG for cube grains remains valid however not with respect to the $S$-component in the deformation texture but with respect to $R$-component (123) [412] .

\section{Results and discussion}

The calculations are performed for recrystallization texture component i.e. cube $(001)\langle 100\rangle$ and deformation texture components, $S$-component (123) [63 $\overline{4}]$ and $R$ component i.e. (123)[41 $\overline{2}]$ in rolled and recrystallized aluminium.

Regarding the rival theories of $\mathrm{ON}$ and $\mathrm{OG}$, evidence has been obtained by Hjelen et al (1991) and Doherty et al (1993) that $\mathrm{ON}$ is operative in the development of cube recrystallization texture in commercial purity aluminium.

As regards the nucleation of cube grains, the classic model of Dillamore and Katoh (1974) with cube grains nucleating in transition bands and the model of Ridha and Hutchinson (1982) where it is proposed that cube subgrains have a lower dislocation density, has been opposed by the microgrowth model of Duggan et al (1995) where the classic OG model for cube texture development have been applied to the cube subgrains with a $40^{\circ}$ [111] with the $S$-subgrain.

In order to clarify the misorientation relationship, this paper addresses the calculation by stereographic projections and analytical calculations.

Figure 1 shows the stereographic projection of (111) poles of an exact cube grain i.e. (001) [100], $S$-component (123) $\langle 63 \overline{4}\rangle$ and $R$-component i.e. (123) $\langle 41 \overline{2}\rangle$.

Figure 1 also shows the axis/angle pair calculation by the Euler method as quoted by Johari and Thomas (1969) on a stereographic projection using (111) poles. The misorientation between cube (001) [100] and $S$-component (123) $[63 \overline{4}]$ comes to about $48^{\circ}$ about an common rotation axis of (111). This result itself shows that $S$-component (123) [634] cannot be a factor for the proposal of OG which dictates a high mobility boundary i.e. $40^{\circ}(111)$. The misorientation between cube (001) [100] and $R$ component (123)[41 $\overline{2}]$ turns out to be $40^{\circ}$ (111) which supports the OG hypothesis with a high mobility boundary supporting the view that cube grains can grow faster if they are associated with the $R$-component of the deformation texture.

Further analytical analysis was carried out and the same result ensued.

The euler angles for an crystallographic orientation is given by Bunge (1982) as follows

$$
\begin{aligned}
& \Phi=\arccos \frac{1}{\sqrt{h^{2}+k^{2}+l^{2}}}, \\
& \phi_{2}=\arcsin \frac{h}{\sqrt{h^{2}+k^{2}}}, \\
& \phi_{1}=\arcsin \frac{w}{\sqrt{u^{2}+v^{2}+w^{2}}},
\end{aligned}
$$

where $\phi_{1}, \phi_{2}, \Phi$ are the 3 euler angles that describe a crystallographic orientation.

For the $R$-component $(123)\langle 41 \overline{2}\rangle$ ideal orientation of the deformation texture, by using (3)-(5), the euler angles are $\phi_{1}=46.729^{\circ}, \phi_{2}=26.56^{\circ}$ and $\Phi=36.6^{\circ}$.

For the $S$-component (123) $\langle 63 \overline{4}\rangle$ of the deformation texture, the euler angles from (3)-(5) are $\phi_{1}=56^{\circ}, \phi_{2}=$ $26 \cdot 56^{\circ}$ and $\Phi=36 \cdot 6^{\circ}$.

The euler angles of the cube orientation (100) $\langle 001\rangle$ are $\phi_{1}=0, \phi_{2}=0$ and $\Phi=0$.

Randle (1992) has given the orientation matrix of an ideal orientation as

$$
T=\left[\begin{array}{lll}
a_{11} & a_{12} & a_{13} \\
a_{21} & a_{22} & a_{23} \\
a_{31} & a_{32} & a_{33}
\end{array}\right],
$$

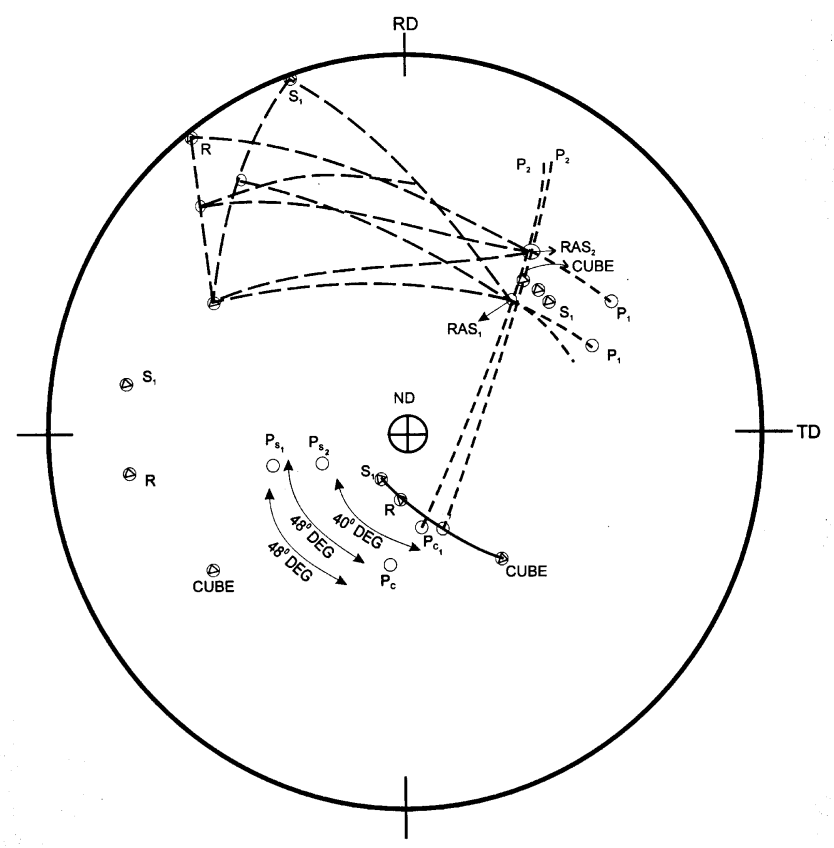

$\left.\begin{array}{l}\text { Deformation Texture } \\ \text { Components }\end{array}\right\} \begin{aligned} & S 1-(123)<63 \overline{4}> \\ & R-(123)<41 \overline{2}>\end{aligned}$

Misorientation $\quad\} \begin{aligned} & \text { S1 \& Cube }-\theta=48^{\circ} \text { about close to }<111> \\ & \text { R \& Cube }-\theta=40^{\circ} \text { about }<111>\end{aligned}$

Figure 1. Exact cube orientation, deformation texture components $S$ and $R$, orientation and misorientation between $S, R$ and cube. 
where $a_{\mathrm{ij}}, \mathrm{i}=1,2,3, \mathrm{j}=1,2,3$ are the direction cosines of the orientation w.r.t. the cube orientation.

Further elements of the matrix are related to the euler angles as follows

$$
\begin{aligned}
& a_{11}=\cos \phi_{1} \cos \phi_{2}+\sin \phi_{1} \sin \phi_{2}, \\
& a_{12}=\sin \phi_{1} \cos \phi_{2}+\cos \phi_{1} \sin \phi_{2} \cos \Phi, \\
& a_{13}=-\sin \phi_{2} \sin \Phi, \\
& a_{21}=-\cos \phi_{1} \sin \phi_{2}-\sin \phi_{1} \cos \phi_{2} \cos \Phi, \\
& a_{22}=\sin \phi_{1} \sin \phi_{2}+\cos \phi_{1} \cos \phi_{2} \cos \Phi, \\
& a_{23}=\cos \phi_{2} \sin \Phi, \\
& a_{31}=\sin \phi_{1} \sin \Phi, \\
& a_{32}=-\cos \phi_{1} \sin \Phi, \\
& a_{33}=\cos \Phi .
\end{aligned}
$$

Based on (6)-(14), the orientation matrix for the $S$ component $(123)\langle 63 \overline{4}\rangle$ is

$$
T_{S}=\left[\begin{array}{rrr}
0.768 & -0.582 & 0.267 \\
0.384 & 0.753 & 0.535 \\
-0.512 & -0.308 & 0.802
\end{array}\right] \text {. }
$$

The orientation matrix for the $R$-component

$$
T_{R}=\left[\begin{array}{cll}
0.872 & 0.896 & 0.266 \\
-0.2199 & 0.815 & 0.532 \\
0.434 & 0.4 & 0.8
\end{array}\right] \text {. }
$$

Randle (1992) has also given the misorientation angle, $\theta$ with exact cube to be expressed as follows

$$
\cos \theta=\frac{a_{11}+a_{22}+a_{33}-1}{2},
$$

and the rotation angle axis by

$$
\begin{aligned}
& 1_{1}=a_{23}-a_{32}, \\
& 1_{2}=a_{31}-a_{13}, \\
& 1_{3}=a_{12}-a_{21},
\end{aligned}
$$

where $\left(1_{1}, l_{2}, l_{3}\right)$ are the indices of the rotation axis.

Using (15)-(16), the misorientation of the $S$-component to the cube component is $48.58^{\circ}$ w.r.t. $[0.843,-0.779$, $-0.966]$ which is close to $\langle 111\rangle$.

Again the misorientation between $R$-component and cube component is $41.9^{\circ}$ about $[0.932,0.7,0.6257]$ which is also close to $\langle 111\rangle$.

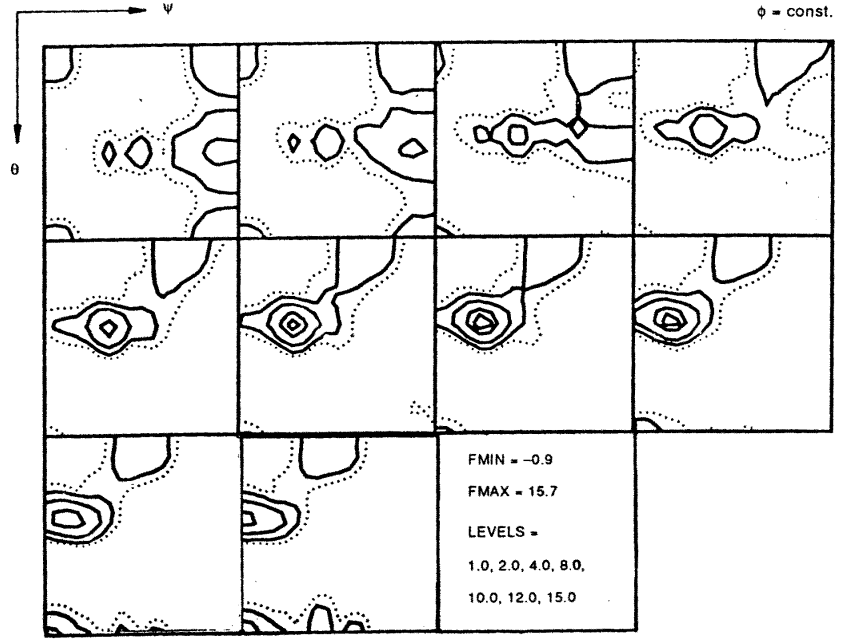

Figure 2. Orientation distribution function (ODF) of commercial purity aluminium showing $S$ and $R$ components of the deformation texture.

It is again seen from the above calculation that it is the $R$-component (123)[41 $\overline{2}]$ which is misoriented from the cube component $(100)\langle 001\rangle$ by $40^{\circ}$ about $\langle 111\rangle$ axis and not the $S$-component (123) $\langle 63 \overline{4}\rangle$.

But $R$-component is present in the deformation texture as shown by Kashyap (1992) in the ODF of the deformation texture in figure 2 for extruded commercial purity aluminium.

Therefore the OG theory is valid for the cube texture development when it is reckoned with the $R$-component of the deformation texture and not the $S$-component.

\section{Conclusions}

It is shown that the $R$-component is misoriented from cube by $40^{\circ}$ about (111) axis and not the $S$-component and that oriented growth theory has to be reckoned with the $R$ component in the development of the cube grains.

\section{Acknowledgement}

The author wishes to thank the management of the Hindustan Aeronautics Ltd., Bangalore, for supporting this work.

\section{References}

Aust K T and Rutter J W 1965 Acta Metall. 13181 Barett C S I 1940 Trans. Am. Soc. Metals 137128

Beck P A and Hu H 1966 in Recrystallization grain growth and textures (Metals Park, Ohio: Am. Soc. Metals)

Bunge H J 1982 Texture analysis in materials science (London: Butterworths)

Burgers W G and Louwerse P C 1931 Z. Phys. 61605

Dillamore I K and Katoh H 1974 Metal. Sci. 873 
Doherty R D, Kashyap K T and Panchanadeeswaran S 1993 Acta Metall. 413029

Duggan B J, Lucke K, Kohlhoff G and Lee C S 1995 Acta Metall. 411921

Grewen J and Huber J 1978 in Recrystallization of metallic materials (ed.) F Haaesner (Stuttgart: Dr Riederey) p. 111 Hjelen J, Ursund R and Nes E 1991 Acta Metall. 39137

Ibe G and Lucke K 1968 in Recrystallization grain growth and textures (Metals Park, Ohio: Am. Soc. Metals) p. 434

Johari O and Thomas G 1969 in Stereographical projection and its applications, Techniques of metals research (ed.) R F Bunshah (New York: Interscience Publishers) Vol. 11A
Kashyap K T 1992 Evolution of recrystallization textures in an aluminium alloy, Ph.D. Thesis, Drexel University, Philadelphia, USA

Lucke K I 19847 Int. conf. textures of materials (Holland: The Netherland Society for Materials Science) p. 195

Mackenzie M K and Thompson M J 1957 Biometrika 44 205

Randle V 1992 Microtexture determination and its applications (London: Institute of Materials)

Ridha A H and Hutchinson W B 1982 Acta Metall. 30 1929

Samajdhar I and Doherty R D 1994 Scr. Metall. 31527 